\title{
AMALGAMATED SUMS OF GROUPS
}

\author{
by J. M. CORSON
}

(Received 15th December 1994)

\begin{abstract}
Groups called amalgamated sums that arise as inductive limits of systems of groups and injective homomorphisms are studied. The problem is to find conditions under which the groups in the system do not collapse in the limit. Such a condition is given by J. Tits when certain subsystems are associated to buildings. This condition can be phrased to apply to certain systems of abstract groups and injective homomorphisms. It is shown to imply that no collapse occurs in the limit in a strong sense; namely the natural homomorphism of the amalgamated sum of any subsystem into the amalgamated sum of the full system is injective. This answers a question of S. J. Pride.
\end{abstract}

1991 Mathematics Subject Classification: Primary 20F05, 20F06; Secondary 20 E99.

\section{Introduction}

We consider amalgams of the following form; our terminology and notation is essentially that of J. Tits [11]. Let $I$ be a set. Assume given (1) for any subset $J \subset I$ of cardinality $|J| \leqq 2$, a group $P_{J}$, (2) for each $J \subset J^{\prime} \subset I$ with $\left|J^{\prime}\right| \leqq 2$, an injective homomorphism $\phi_{J J^{\prime}}: P_{J} \rightarrow P_{J^{\prime}}$, such that $\phi_{J J}=$ id and $\phi_{J J^{\prime \prime}}=\phi_{J^{\prime} J^{\prime \prime}} \phi_{J J^{\prime}}$ for $J \subset J^{\prime} \subset J^{\prime \prime}$. We often write $P_{\emptyset}=B, P_{\{i\}}=P_{i}, \phi_{\emptyset[i\}}=\phi_{i}$, and $P_{\{i, j\}}=P_{i j}=P_{j i}$ for $i, j \in I$. In addition, we shall require that (3) for each $i, j \in I, P_{i j}$ is generated by the images of $P_{i}$ and $P_{j}$.

This note concerns the 'crucial problem' of finding conditions under which the inductive limit $G$, also called the amalgamated sum, of such an amalgam $P$ does not collapse. It is perhaps worth noting that $G$ can be viewed as the free product of the $P_{J}$ (for $|J| \leqq 2$ ) with added relations $\phi_{J J}(x)=x$ for all choices of $J, J^{\prime}$, and $x$ (such that $J \subset J^{\prime},\left|J^{\prime}\right| \leqq 2$, and $x \in P_{J}$ ).

For distinct $i, j \in I$, observe that the injective homomorphisms $P_{i} \rightarrow P_{i j}$ and $P_{j} \rightarrow P_{i j}$ determine a homomorphism $P_{i}{ }_{B} P_{j} \rightarrow P_{i j}$. Let $2 m_{i j}$ be the length of a shortest nontrivial element in its kernel (in the usual length function on the amalgamated free product), or put $m_{i j}=\infty$ if the kernel is trivial. When such a nontrivial word exists, it has even length so $m_{i j}$ is a positive integer or $\infty$.

Three distinct elements $i, j, k \in I$ shall be called a spherical triple with respect to $P$ if

$$
\frac{1}{m_{i j}}+\frac{1}{m_{j k}}+\frac{1}{m_{k i}}>1
$$

(where $1 / \infty$ is understood to mean 0 ). In [8, p. 210], S. J. Pride remarks that it seems 
plausible that if no spherical triples with respect to $P$ exist, then all the natural homomorphisms of the given groups $P_{i j}$ into the amalgamated sum $G$ are injective. Our aim is to prove this result. In fact, we prove more, namely the theorem below.

For $K \subset I$, let $G_{K}$ be the amalgamated sum of the system of groups $\left\{P_{J}: J \subset K\right.$ and $|J| \leqq 2\}$ relative to the set of homomorphisms $\left\{\phi_{J J^{\prime}}: J \subset J^{\prime} \subset K\right.$ and $\left.\left|J^{\prime}\right| \leqq 2\right\}$. In particular $G_{I}=G$.

Theorem. Suppose I contains no spherical triples with respect to $P$. Then for each subset $K \subset I$, the natural homomorphism $G_{K} \rightarrow G$ is injective.

Remarks. 1. In the case where $|I|=3$, such an amalgam $P$ has been termed a triangle of groups. The theorem for triangles of groups has been shown by Gersten and Stallings; see [10]. For generalizations in another direction, to complexes of groups, see also $[2,3,5,9]$.

2. Some special cases of the theorem are established by Pride; see [7, Theorem 4] and [8, Theorem 3]. Edjvet [4] extends Pride's results to more general types of amalgams than those considered here.

3. In the case that the chamber system associated to each subamalgam on a subset $J \subset I$ with $|J|=2$ is a building, the theorem follows from an amazing result of $J$. Tits [11, Theorem 1]. In this case much more is true, namely the chamber system associated to the full amalgam is a building. Furthermore, Tits does not assume that no spherical triples exist, he only requires that the chamber system associated to a spherical triple be a building.

4. The theorem remains true without the requirement (3) that each $P_{i j}$ be generated by the images of $P_{i}$ and $P_{j}$, however, the arguments become more complicated.

The methods we use are geometric, employing the notion of a picture; recall that pictures are dual to van Kampen diagrams [6, Chapter V]. The alternative statement of the theorem below is more suitable for this technique of proof. We first introduce some notation: For each subset $K \subset I$, let $F_{K}$ denote the free product of the family $\left\{P_{k}\right\}_{k \in K}$ with the subgroup $B$ amalgamated via the monomorphisms $\phi_{k}: B \rightarrow P_{k}$. The elements of $F_{K}$ are represented (non-uniquely) by words $a_{1} \ldots a_{n}$ whose letters $a_{i}$ belong to $\cup_{k \in K} P_{k}$ (disjoint union).

Condition (3) implies that for $K \subset I$, the group $G_{K}$ is generated by the images of the groups $P_{k}, k \in K$. Thus, the homomorphism $F_{K} \rightarrow G_{K}$ determined by the natural homomorphisms $P_{k} \rightarrow G_{K}$ is surjective. In particular, for distinct $i, j \in I$, the homomorphism $P_{i} *_{B} P_{j} \rightarrow P_{i j}$ is surjective; let $R_{i j}=R_{j i}$ be the set of words representing elements of its kernel.

Put $R_{K}=\cup R_{i j}$ where the union is over the distinct pairs $i, j \in K$. Let $N_{K}$ be the normal subgroup of $F_{K}$ generated by $R_{K}$. Then $N_{K}$ is the kernel of the natural homomorphism $F_{K} \rightarrow G_{K}$; whence $F_{K} / N_{K} \cong G_{K}$. This can be seen by observing that the group $F_{K} / N_{K}$ and natural homomorphisms $P_{J} \rightarrow F_{K} / N_{K}$ for $J \subset K$ and $|J| \leqq 2$ possess the universal mapping property defining the amalgamated sum of the amalgam restricted to $K$ (see [11]).

Set $F_{I}=F, R_{I}=R$ and $N_{I}=N$; thus $G \cong F / N$ where $N$ is the normal subgroup 
generated by $R$. Now the theorem is precisely the Freiheitssatz: The inclusion induced homomorphism $F_{K} / N_{K} \rightarrow F / N$ is injective for each subset $K \subset I$.

\section{Preliminaries}

Disc pictures. A picture $\Gamma$ consists of a finite disjoint union of (small) discs $v_{1}, \ldots, v_{n}$ in the interior of the standard closed disc $D^{2}$, and a compact 1-manifold $\xi$ properly embedded in $\Sigma=D^{2} \backslash \operatorname{Int}\left(\cup v_{i}\right)$; the following terms shall be employed:

vertices: the embedded discs $v_{1}, \ldots, v_{n}$

arcs: the components of $\xi$

corners: the components of $\partial \Sigma \backslash \xi$ (excluding those that are circles)

regions: the components of $D^{2} \backslash \Gamma$.

A region $\Delta$ is called interior if it does not meet $\partial D^{2}$; otherwise $\Delta$ is called a boundary region. A picture is termed spherical if it has at least one vertex and no arcs meeting $\partial D^{2}$. Observe that a spherical picture has exactly one boundary region. (See [1] for a survey of the theory of pictures.)

The degree or valence of a vertex $v$ of a picture $\Gamma$, denoted by $d(v)$, is the number of arcs incident with $v$, counted with multiplicity so that an arc with two endpoints on $v$ contributes 2 to $d(v)$; equivalently, $d(v)$ is the number of corners in the boundary of $v$. The degree of a region $\Delta$ of $\Gamma$, denoted by $d(\Delta)$, is the number of arcs in the boundary of $\Delta$, counted with multiplicity so that an arc with $\Delta$ on both sides contributes 2 to $d(\Delta)$; equivalently, $d(\Delta)$ is the number of corners in the boundary of $\Delta$.

A corner weighting function on a picture $\Gamma$ is a function $\theta$ assigning a real number (called an angle) to each corner of $\Gamma$. Given a vertex $v$ of $\Gamma$, let $\theta(v)$ denote the sum of the weights on the corners of $v$. Similarly, for each region $\Delta$, let $\theta(\Delta)$ denote the sum of weights on the corners in $\partial \Delta$.

We record the following immediate consequence of the curvature formula for spherical pictures; see for example [1, p. 159].

Lemma 2.1. Suppose $\Gamma$ is a connected spherical picture and let $\theta$ be a corner weighting function satisfying:

(i) $\theta(v) \geqq 2 \pi$ for each vertex $v$;

(ii) $\theta(\Delta) \leqq[d(\Delta)-2] \pi$ for each interior region $\Delta$.

Then the unique boundary region $\Delta_{\infty}$ is such that $\theta\left(\Delta_{\infty}\right) \geqq\left[d\left(\Delta_{\infty}\right)+2\right] \pi$.

Coloured pictures. Now let $I$ be a fixed set whose elements shall be referred to as colours, and let $\left\{m_{i j}\right\}_{i, j \in I}$ be a fixed family of elements of $\mathbb{N} \cup\{\infty\}$ such that $m_{i j}=m_{j i}$ and for $i \neq j, m_{i j} \geqq 2$.

We make use of the following terminology: a triple of distinct colours $i, j, k \in I$ shall be called a spherical triple if

$$
\frac{1}{m_{i j}}+\frac{1}{m_{j k}}+\frac{1}{m_{k i}}>1
$$


(where $1 / \infty$ is understood to mean 0 ).

By a colouring of a picture $\Gamma$ by $I$ we mean an $I$-valued function on the set of arcs of $\Gamma$; a picture together with a colouring function into $I$ shall be called an $I$-coloured picture.

Lemma 2.2. Suppose I contains no spherical triples and let $\Gamma$ be an I-coloured picture satisfying:

(1) No arc is a circle, nor has both endpoints on the same vertex enclosing a region of degree one.

(2) Associated to each vertex $v$ are two distinct colours $i, j \in I$ (with $m_{i j} \neq \infty$ ) such that each arc incident with $v$ is coloured either $i$ or $j$ and there are at least $2 m_{i j}$ corners of $v$ joining one arc of each colour.

(3) No interior region has more than one corner in its boundary joining arcs of the same two distinct colours.

Conclusion: If some arc of $\Gamma$ is coloured $i_{0}$, then some arc meeting $\partial D^{2}$ is coloured $i_{0}$.

Proof. Suppose some arc of $\Gamma$ is coloured $i_{0}$, but no arc meeting $\partial D^{2}$ is coloured $i_{0}$. Let $\Gamma^{\prime}$ be a connected component of the $i_{0}$-skeleton of $\Gamma$ (subpicture consisting of the arcs coloured $i_{0}$ and the vertices incident with such an arc). By assumption, $\Gamma^{\prime}$ is a spherical picture; let $\Delta_{\infty}$ be its unique boundary region. Now let $\Gamma_{0}$ be a connected component of the picture obtained from $\Gamma$ by deleting the vertices and interiors of arcs that lie in $\Delta_{\infty}$. Then $\Gamma_{0}$ is a connected spherical picture with boundary region $\Delta_{\infty}$, and all the arcs in $\partial \Delta_{\infty}$ are coloured $i_{0}$.

Define a corner weighting function $\theta$ on $\Gamma_{0}$ as follows: let $\alpha$ be a corner in the boundary of an interior region of $\Gamma_{0}$. If $\alpha$ joins one arc coloured $i$ and one arc coloured $j$, we assign the angle $\theta(\alpha)=\pi / m_{i j}$ if $i \neq j$ and $\theta(\alpha)=0$ if $i=j$. The remaining corners-those contained in $\partial \Delta_{\infty}$-are given the angle $\pi$. With these angles, we shall observe that the hypotheses (i) and (ii) of Lemma 2.1 hold for $\Gamma_{0}$.

For vertices not in $\partial \Delta_{\infty}$, condition (i) follows immediately from (2) and the definition of $\theta$. Suppose $v$ is vertex in $\partial \Delta_{\infty}$. Then $v$ meets arcs coloured $i_{0}$, and by (2), $v$ meets arcs of precisely two colours; say $j$ is the other colour. Since all $i_{0}$-coloured arcs of $\Gamma$ that meet $v$ also belong to $\Gamma_{0}$ (as $\Gamma_{0}$ contains a component of the $i_{0}$-skeleton of $\Gamma$ ), it follows by (2), that $v$ contains at least $2 m_{i_{0} j}-2$ corners of $\Gamma_{0}$ having angle $\pi / m_{i_{0} j}$. Also $v$ contains at least one corner with angle $\pi$; whence condition (i) holds for $v$ (recall that $m_{i \mathrm{ioj}} \geqq 2$ ).

To verify condition (ii), let $\Delta$ be an interior region of $\Gamma_{0}$. Initially, it should be noted that $d(\Delta) \neq 1$, by (1), so the right hand side of the inequality (ii) is at least zero. If the arcs in $\partial \Delta$ are all the same colour, then $\theta(\Delta)=0$ and the condition holds. It is not possible for the arcs in $\partial \Delta$ to be of exactly two distinct colours by (3). Suppose $\partial \Delta$ consists of arcs of exactly three distinct colours, say $i, j, k \in I$. Then by (3), there are exactly three corners in $\partial \Delta$ joining arcs of different colours. Since $I$ contains no spherical triples,

$$
\theta(\Delta)=\frac{\pi}{m_{i j}}+\frac{\pi}{m_{j k}}+\frac{\pi}{m_{k i}} \leqq \pi
$$


Also $d(\Delta) \geqq 3$ so (ii) holds for such regions. Finally, if there are arcs in the boundary of $\Delta$ of at least four distinct colours, then $d(\Delta) \geqq 4$ and (ii) automatically holds since the corner weights are at most $\pi / 2$ (as each $m_{i j} \geqq 2$ ). This completes the verification of (ii).

Invoking Lemma 2.1, we conclude that

$$
\theta\left(\Delta_{\infty}\right) \geqq\left[d\left(\Delta_{\infty}\right)+2\right] \pi .
$$

But it follows directly from the definition of $\theta$ that $\theta\left(\Delta_{\infty}\right)=\pi d\left(\Delta_{\infty}\right)$, a contradiction.

Pictures over $\langle F \mid R\rangle$. Let $F$ and $R$ be as in the introduction. By a picture over $\langle F \mid R\rangle$ we shall mean a picture $\Gamma$ together with the additional data:

(0) Each arc is given a transverse orientation and is labelled by an element of $\cup_{i \in I} P_{i}$ (disjoint union).

(1) The sequence of labels on the arcs meeting a vertex $v$, in order once around the boundary of $v$, gives a word in $R$. (Of course, this condition is independent of the direction and starting point.)

A Picture over $\langle F \mid R\rangle$ is given the obvious colouring (by $I$ ): arcs labelled by elements of $P_{i}$ are coloured $i$.

A boundary label of $\Gamma$ is, by definition, a word obtained by reading the labels on the arcs meeting $\partial D^{2}$, in order once around $\partial D^{2}$ in some direction. Recall that the boundary label is a consequence of the words on the vertices, i.e., the element of $F$ represented by a boundary label is contained in the normal closure of the elements represented by the words around the vertices.

Let $w$ be an element of $F$. By a picture of $w$, we shall mean a picture $\Gamma$ over $\langle F \mid R\rangle$ such that some boundary label of $\Gamma$ is a word representing $w$. A picture of $w$ is termed minimal if (a) no arc is a circle, nor has both endpoints on the same vertex enclosing a region of degree one, and (b) no picture of $w$ has fewer vertices than $\Gamma$. (An arc such as in (a), together with the components of the picture it encloses, can always be removed producing a simpler picture of $w$.)

Remark 2.3. A minimal picture $\Gamma$ of $w$ satisfies the three hypotheses of Lemma 2.2: (1) This is assumed by the definition of minimal. (2) This follows from the definition of the $m_{i j}$ upon noting that no word labelled around a vertex of $\Gamma$ represents the trivial group element: if this situation occurred, then it is a well-known fact that there would be a picture for $w$ with one less vertex. (3) Assume that such an interior region in $\Gamma$ exists; let $\alpha$ and $\beta$ be two corners in its boundary joining arcs of the same two distinct colours, say $i$ and $j$. There are two cases to consider:

Firstly, suppose $\alpha$ and $\beta$ lie on distinct vertices of $\Gamma$, say $v_{1}$ and $v_{2}$. Take a simple closed curve $C$ enclosing only the vertices $v_{1}$ and $v_{2}$, and having only transverse intersections with arcs (see Fig. 1). Observe that the product of the labels encountered in a trip around $C$ is an element of $R_{i j}$ Hence, replacing $v_{1}$ and $v_{2}$ with a single vertex (with boundary $C$ ), we get a picture for $w$ with one less vertex - a contradiction.

Secondly, suppose $\alpha$ and $\beta$ are on the same vertex, say $v$. Then the region is as depicted in Fig. 2; we draw a simple closed curve $C$ as shown. The subpicture bounded 


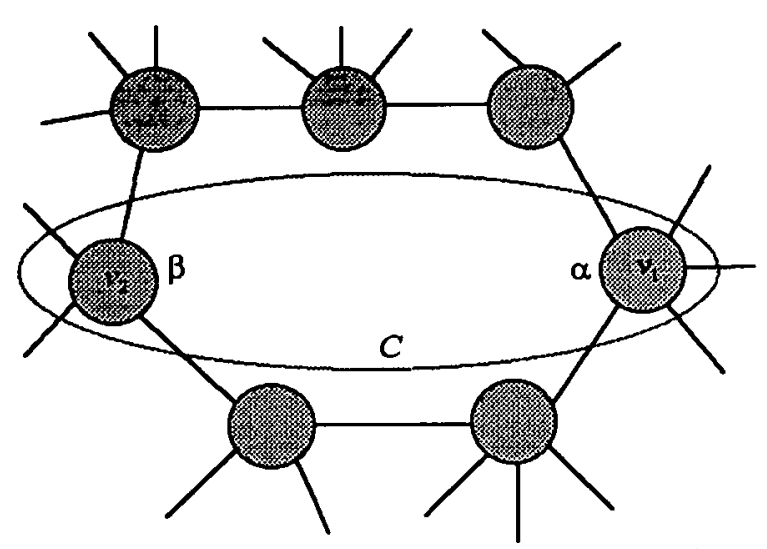

FIGURE 1

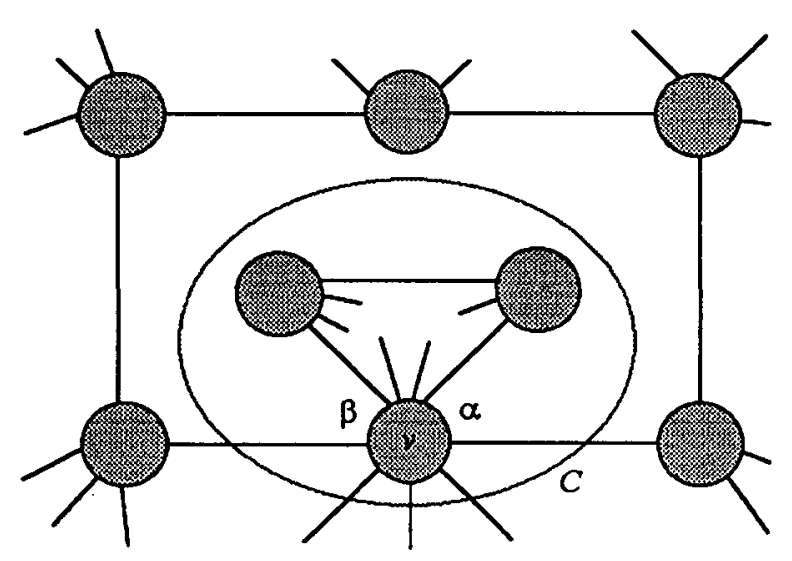

FIGURE 2

by $C$ contains at least one vertex in addition to $v$, so that condition (1) is not violated. By assuming our region is an 'innermost' one violating condition (3) (meaning that condition (3) is satisfied by every region that is enclosed by a simple closed 'path' consisting of vertices and arcs of $\Gamma$ that lie in the boundary of our region), we may assume that Lemma 2.2 applies to the subpicture bounded by $C$. Hence, each arc in the subpicture is coloured $i$ or $j$, as only arcs of these colours meet its boundary. It follows that a boundary label of the subpicture is also a word in $R_{i j}$ (as, by its definition, $R_{i j}$ contains all of its consequences). Now everything enclosed by $C$ can be replaced by a single vertex, thus producing a picture for $w$ with fewer vertices than $\Gamma$. Again this contradicts minimality. 
From the standpoint of the considerations in Section 3, the following observation is of key importance:

Proposition 2.4. Suppose I contains no spherical triples and let $w$ be a cyclically reduced element of $F$. Then $w \in N$ if and only if there exists a minimal picture of $w$ with a boundary label which is a cyclically reduced word representing $w$.

This is rather easily proved by appropriately modifying the standard procedure of producing pictures over 2-complexes with analogous properties. However, there is a subtle point involved in achieving a cyclically reduced boundary label; indeed the proposition is probably not true in general without some type of curvature assumption. One should expect this due to the same problem arising in the dual situation of van Kampen diagrams labelled over free products with amalgamation; see [6]. We sketch a proof of the proposition in an appendix.

\section{The proof}

We are now in a position to supply the details of the proof outlined in the introduction. Initially, we consider the special case: each $m_{i j} \geqq 2$ for $i \neq j$. (Recall that this condition is assumed in Lemma 2.2.)

Fix $K \subset I$. Let $w$ be a cyclically reduced word in $F_{K}$ representing an element in the kernel of the natural homomorphism $F_{K} \rightarrow F / N$. Then by regarding $w$ as an element of $F$, via the obvious inclusion $F_{K} \hookrightarrow F$, there is a minimal picture $\Gamma$ of $w$ with a boundary label which is a cyclically reduced word representing $w$ (by Proposition 2.4). The key observation here is that each arc of $\Gamma$ that meets $\partial D^{2}$ is coloured by an element of $K$, as a cyclically reduced word representing an element of $F_{K}$ only contains letters from the $P_{k}$ (for $k \in K$ ). Since the hypothesis of Lemma 2.2 holds for minimal pictures (as noted in Remark 2.3), we conclude that every arc in $\Gamma$ is coloured by an element of $K$. Thus $w \in N_{K}$, the normal closure of $R_{K}$ in $F_{K}$. Consequently, the kernel of $F_{K} \rightarrow F / N$ is $N_{K}$, completing the proof in this case.

For the general case, consider the simplicial graph whose vertex set is $I$ and whose edge set consists of the pairs $(i, j)$ such that $m_{i j} \neq \infty$. The vertex sets of the connected components of this graph form a partition of the set $I$, say $\left\{I_{\lambda}\right\}_{\lambda \in \Lambda}$. Now the theorem follows from the special case, and well-known properties about free products with an amalgamated subgroup, once we establish the claim: $G$ is the free product of the family of groups $\left\{G_{I_{\lambda}}\right\}_{\lambda \in \Lambda}$ with the subgroup $B$ amalgamated, and the theorem holds for the restriction of the amalgam $P$ to each subset $I_{\lambda}$.

The second assertion follows from the special case unless $I_{\lambda}$ contains distinct indices $i, j$ such that $m_{i j}=1$. But this implies that $I_{\lambda}=\{i, j\}$; otherwise $I_{\lambda}$ would contain a spherical triple. The theorem holds automatically for restrictions to such subsets.

If $i$ and $j$ do not belong to the same subset $I_{\lambda}$, then $m_{i j}=\infty$; whence every word in $R_{i j}$ represents the identity. Hence, the first assertion is seen by viewing $G$ as the quotient of $F=*_{B} P_{i}$ by the normal subgroup generated by $R=\cup R_{i j}$. (The natural homomorphism 

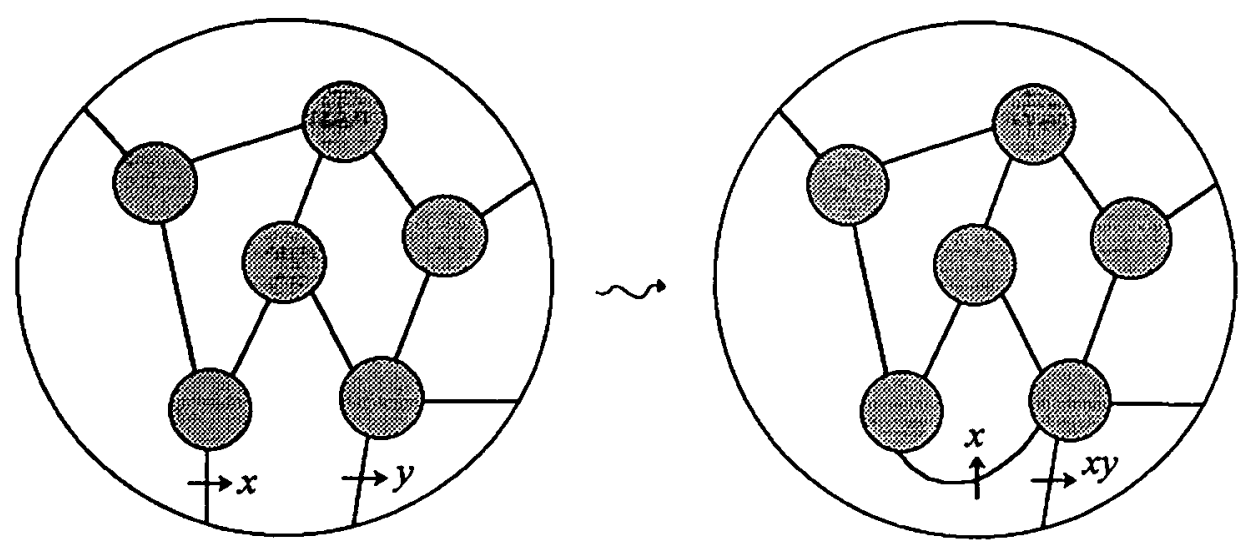

FIGURE 3

$B \rightarrow G_{I_{\lambda}}$ is injective by definition if $\left|I_{\lambda}\right| \leqq 2$, and by the special case applied to the restriction of the amalgam $P$ to the subset $I_{\lambda}$ if $\left|I_{\lambda}\right| \geqq 3$.)

\section{Appendix: proof of proposition 2.4}

First of all, it is clear that minimal pictures of $w$ exit, for example, the picture dual to any least area van Kampen diagram for $w$-such diagrams are exhibited in [6, Chapter V]. The nontrivial part of the proposition is that we can find such a picture with a cyclically reduced boundary label. To accomplish this, we start with any minimal picture $\Gamma$ of $w$ and apply various moves, having the effect of cyclically reducing the boundary label.

I. Consolidation move. Suppose successive arcs encountered in a boundary cycle are labelled by elements $x$ and $y$ in the same group $P_{i}$. Then this move, as illustrated in Fig. 3 , results in one arc meeting the boundary and labelled by the product $x y$.

II. Arc deleting move. Delete the interior of an arc labelled by the trivial element of some $P_{i}$.

III. Relabelling move. Let $\Delta$ be a region of $\Gamma$ and $b \in B$. Then the arcs in $\partial \Delta$ are relabelled as follows: let $e$ be such an arc and suppose the label on $e$ is $x \in P_{i}$. If $\Delta$ is on just one side of $e$, then replace the label by $x \phi_{i}(b)$ or $\phi_{i}(b)^{-1} x$ depending as the transverse direction points inward or outward from $\Delta$. If $\Delta$ is on both sides of $e$, replace the label on $e$ by $\phi(b)^{-1} x \phi_{i}(b)$. In the example shown in Fig. 4, initially the arcs were labelled by the $x_{i}$, and $b_{i}=\phi_{j_{i}}(b)$ where $j_{i}$ is the colour of the arc initially labelled $x_{i}$.

Under our assumption of no spherical triples, the last possibility in the relabelling move actually does not occur:

Lemma. If $\Gamma$ is a minimal picture of $w$, then no arc of $\Gamma$ has the same region on both sides. 


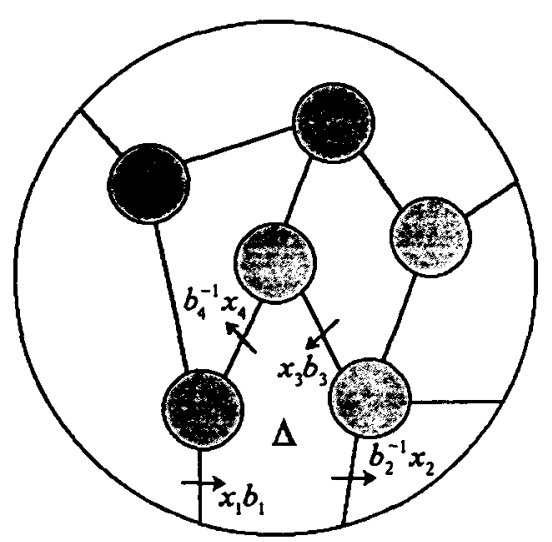

FIGURE 4

Proof. Suppose $e$ were such an arc, say with the region $\Delta$ on both sides. Then there exists a simple closed curve that lies in $\Delta$ except at one point where it meets $e$ transversely. But this is impossible in a minimal picture by the analogous argument used in Remark 2.3.

Note the use of the lemma in the following process: Suppose $\Gamma$ is a minimal picture of $w$; let $a_{1} \ldots a_{n}$ be a boundary label representing $w$. If successive letters $a_{i}$ and $a_{i+1}$ (indices modulo $n$ ) belong to the same $P_{j}$, then there is a consolidation move having the effect of replacing the two letters by a single letter-their product in $P_{j}$. Suppose some letter $a_{i}$ is in the image of $B$. If $e$ is the arc with label $a_{i}$, then there is a region that lies on just one side of $e$ (by the lemma). Performing a relabelling move to this region, we can change the label on $e$ to 1 . Then the arc $e$ can be removed by an arc deleting move.

After finitely many steps, this process stops with a picture whose boundary labels are cyclically reduced words. The boundary label starting at the point that initially gave the word $a_{1} \ldots a_{n}$, now represents an element of $F$ that differs from $w$ at most by conjugation by an element of $B$. Applying a relabelling move, we can arrange that some boundary label is a cyclically reduced word representing $w$, precisely.

\section{REFERENCES}

1. W. Bogley and S. J. PRIDE, Calculating generators of $\pi_{2}$, in Two-dimensional homotopy and combinatorial group theory (C. Hog-Angeloni, W. Metzler, A. Sierodsky, eds., London Mathematical Society Lecture Note Series 197, Cambridge University Press, Cambridge, 1993), 157-188.

2. A. Chermak, Non-spherical assemblages and hyperbolic automorphisms of trees, preprint.

3. J. M. Corson, Complexes of groups, Proc. London Math. Soc. (3) 65 (1992), 199-224.

4. M. Edjvet, On a certain class of group presentations, Math. Proc. Cambridge Philos. Soc. 105 (1989), 25-35.

5. A. HAEfliger, Complexes of groups and orbihedra, in Group theory from a geometrical viewpoint (Ghys, Haefliger and Verjovsky, eds., World Scientific, Singapore, 1991), 504-540. 
6. R. C. Lyndon and P. E. SChupP, Combinatorial group theory (Ergebnisse der Mathematik und ihrer Grenzgebiete 89, Springer, Berlin-Heidelberg-New York, 1977).

7. S. J PRIDE, Groups with presentations in which each defining relator involves exactly two generators, J. London Math. Soc. (2) 36 (1987), 245-256.

8. S. J. PRIDE, The (co)homology of groups given by presentations in which each defining relator involves at most two types of generators, J. Austral. Math. Soc. Ser. A 52 (1992), 205-218.

9. B. SPIELER, Ph.D. thesis, The Ohio State University, 1992.

10. J. R. Stallings, Non-positively curved triangles of groups, in Group theory from a geometrical viewpoint (Ghys, Haefliger and Verjovsky, eds, World Scientific, Singapore, 1991), 491-503.

11. J. Tirs, Buildings and group amalgamations, in Proceedings of Groups-St Andrews 1985 (E. F. Robertson and C. M. Campbell, eds., London Mathematical Society Lecture Notes Series 121, Cambridge University Press, Cambridge, 1986), 110-127.

Department of Mathematics

University OF Alabama

Box 870350

Tuscaloosa, AL 35487-0350

U.S.A.

E-mail address: jcorson@mathdept.as.ua.edu 